\title{
The long-term effects of neurotoxic doses of methamphetamine on the extracellular concentration of dopamine measured with microdialysis in striatum
}

\author{
Terry E. Robinson, Jay Yew, Pamela E. Paulson and Dianne M. Camp \\ Department of Psychology and Neuroscience Program, The University of Michigan, Ann Arbor, MI 48109 \\ (U.S.A.)
}

(Received 20 September 1989; Revised version received 26 October 1989; Accepted 31 October 1989)

Key words: Neurotoxicity; Striatum; 5-Hydroxyindoleacetic acid; Dihydroxyphenylacetic acid; Homovanillic acid; (+)-Amphetamine; Microdialysis; Plasticity; Release

The extracellular concentrations of dopamine (DA), dihydroxyphenylacetic acid (DOPAC), homovanillic acid (HVA), and 5-hydroxyindoleacetic acid (5-HIAA) in the striatum were measured by in vivo microdialysis in freely moving rats one week after the animals were treated with neurotoxic doses of methamphetamine. Methamphetamine produced a marked depletion of striatal DA measured in postmortem tissue, and in the extracellular concentrations of DOPAC, HVA and 5-HIAA. In contrast, the resting extracellular concentration of DA in striatum was the same as in saline-pretreated controls. Furthermore, methamphetamine-pretreated rats were able to increase their concentration of extracellular DA to the same extent as controls in response to a $(+)$-amphetamine challenge. It is suggested that this adaptive response is probably responsible, at least in part, for the absence of obvious behavioral deficits in animals exposed to neurotoxic doses of methamphetamine.

Repeated treatment with high doses of methamphetamine is toxic to dopamine (DA) and serotonin neurons (see ref. 14 for review). For example, it causes a longlasting depletion of striatal DA, loss of DA reuptake sites and decrease in tyrosine hydroxylase activity $[9,10]$; effects that are accompanied by degeneration of striatal DA terminals [14]. Despite this long-lasting neuronal damage, methamphetamine pretreatment does not produce any of the deficits in spontaneous behavior typically associated with DA depletion. Rats show normal rates of milk drinking [5, 7], and rhesus monkeys show normal eye tracking function and fine hand coordination $[3,4]$.

Studies in animals depleted of striatal DA with 6-hydroxydopamine (6-OHDA) have shown that functions are spared following a partial 6-OHDA lesion, presuma-

Correspondence: T.E. Robinson, The University of Michigan, Neuroscience Laboratory Bldg., 1103 E. Huron St., Ann Arbor, MI 48109, U.S.A. 
bly because of presynaptic compensatory changes in the remaining terminals [15] that serve to maintain a relatively normal extracellular concentration of DA $[1,6,13]$. The most toxic doses of methamphetamine produce a persistent depletion of striatal DA by no more than about $80-85 \%[9,14]$, and therefore, similar compensatory changes may be responsible for the sparing of function associated with neurotoxic methamphetamine treatment. To test this hypothesis rats were given a series of neurotoxic doses of methamphetamine, and one week later the extracellular concentration of DA in striatum was estimated by use of in vivo microdialysis.

Male Holtzman rats were anesthetized and a stainless-steel guide cannula was placed stereotaxically on the dural surface above the corpus of the neostriatum. Approximately one week following surgery each animal received an injection of 15 $\mathrm{mg} / \mathrm{kg}$ of methamphetamine (s.c.), or saline, once every $6 \mathrm{~h}$ for a total of 5 injections; a treatment previously shown to produce a long-lasting depletion of striatal DA [9]. One week after the last injection each animal was lightly anesthetized with ether and a removable version of the dialysis probe described by Robinson and Whishaw [13] was inserted into the guide cannula and fixed in the location illustrated on Fig. 4 in Robinson and Whishaw [13]. Each animal was then put into a hemispherical test chamber and left undisturbed until the dialysis experiment began the next day. At least 3 samples of dialysate were collected under resting (baseline) conditions, and then each animal received a challenge injection of $1.5 \mathrm{mg} / \mathrm{kg}$ of $(+)$-amphetamine sulfate (weight of the salt; i.p.) and sample collection continued for $200 \mathrm{~min}$. The effect of $(+)$-amphetamine on motor behavior was also quantified. Each animal was decapitated a few days later and tissue obtained for neurochemical analysis.

The dialysis probe and analytical methods were similar to those described in detail elsewhere $[12,13]$. Briefly, the concentric-style dialysis probe had an outside diameter of $250 \mu \mathrm{m}$, a $4 \mathrm{~mm}$-long tip and was perfused with Ringers $(128.3 \mathrm{mM} \mathrm{NaCl}, 2.68$ $\mathrm{mM} \mathrm{KCl}, 1.35 \mathrm{mM} \mathrm{CaCl}_{2}, 2.0 \mathrm{mM} \mathrm{MgCl}_{2}, \mathrm{pH} 7.3$ ) at $1.5 \mu 1 / \mathrm{min}$. Samples were collected over $20 \mathrm{~min}$ intervals. Dialysate was analyzed within $20 \mathrm{~min}$ after collection for DA, dihydroxyphenylacetic acid (DOPAC), homovanillic acid (HVA) and 5-hydroxy-indoleacetic acid (5-HIAA) by high performance liquid chromatography (HPLC), with series oxidative-reductive electrochemical detection using flowthrough 'coulometric' electrodes (ESA, Bedford, MA). All probes were tested for recovery in vitro at $37^{\circ} \mathrm{C}$ prior to implantation, and dialysate values are corrected for probe recovery.

Methamphetamine pretreatment caused a large depletion of DA and serotonin (5-HT) in postmortem tissue, as expected [9]. For example, the postmortem tissue concentration of striatal DA in methamphetamine-pretreated rats (mean \pm S.E.M., $2.7 \pm 0.59 \mathrm{ng} / \mathrm{mg}$ wet tissue wt.) was only $18.5 \%$ of that in saline-pretreated controls $(14.51 \pm 1.06)$. The postmortem tissue concentration of DA in the nucleus accumbens was less affected by methamphetamine pretreatment, with the concentration in methamphetamine-pretreated rats $(5.97 \pm 0.49)$ being $50.9 \%$ of control $(11.73 \pm 0.68)$. 5-HT was also greatly depleted by methamphetamine. For example, in the medial frontal cortex of methamphetamine-pretreated rats 5 -HT was $0.143 \pm 0.04 \mathrm{ng} / \mathrm{mg}$, which was only $20 \%$ of the control levels $(0.713 \pm 0.17)$. 


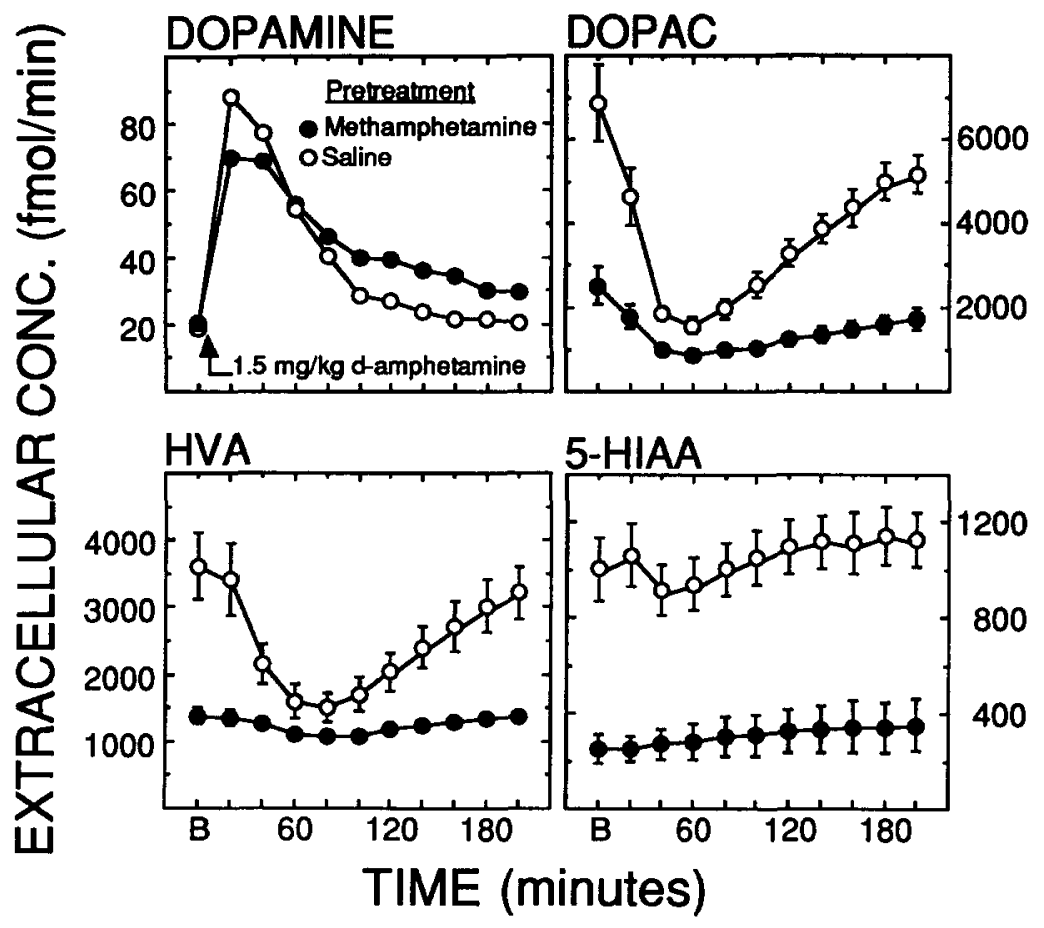

Fig. 1. The average ( \pm S.E.M.) extracellular concentrations ( $\mathrm{fmol} / \mathrm{min}$; corrected for probe recovery) of dopamine, dihydroxyphenylacetic acid (DOPAC), homovanillic acid (HVA) and 5-hydroxyindoleacetic acid (5-HIAA) estimated by microdialysis during baseline (B) and for ten 20 min intervals after a challenge injection of $1.5 \mathrm{mg} / \mathrm{kg}$ of $(+)$-amphetamine. Rats were pretreated with either methamphetamine (solid symbols; $n=5$ ) or saline (open symbois; $n=7$ ). S.E.M. bars are not shown for dopamine because they are overlapping at all points of time. Dopamine: a two-way ANOVA with repeated measures resulted in no significant effect of group, a highly significant effect of time, and a significant group by time interaction $(F=2.69, P<0.008)$. However, the two groups did not differ significantly at any single point in time, including baseline (individual $t$-tests). DOPAC: two-way ANOVA, effect of group ( $F=22.1, P<0.001$ ), time $(F=56.1, P<0.001)$ and group-by-time interaction $(F=14.5, P<0.001)$. The two groups differed at all points in time (individual $t$-tests). HVA: effect of group $(F=9.82, P<0.01)$, time $(F=28.8, P<0.001)$ and group-by-time interaction $(F=12,0, P<0.001)$. The groups differed at all points in time except between 60 and $100 \mathrm{~min}$ after $(+)$-amphetamine (individual $t$-tests). 5-HIAA: effect of group $(F=23.8$, $P<0.001)$, time $(F=8.85, P<0.001)$ and group-by-time interaction $(F=1.95, P<0.05)$. The groups differed at all points in time (individual $t$-tests).

Despite the depletion of striatal DA in postmortem tissue, methamphetamine pretreatment had no effect on the extracellular concentration of striatal DA under resting conditions $(20.22 \pm 5.9$ and $18.69 \pm 3.6 \mathrm{fmol} / \mathrm{min}$ in methamphetamine vs salinepretreated rats, respectively). In contrast, methamphetamine pretreatment depleted resting extracellular DOPAC, HVA and 5-HIAA by $63.5 \%, 61.9 \%$ and $75 \%$, respectively, relative to saline-pretreated animals (Fig. 1).

The challenge injection of $(+)$-amphetamine produced a large increase in the extracellular concentration of DA in both control and methamphetamine-pretreated 
animals (Fig. 1). Although methamphetamine-pretreated rats showed a somewhat attenuated initial response to the $(+)$-amphetamine challenge, this tendency was not quite statistically significant. $(+)$-Amphetamine produced a significant decrease in the extracellular concentration of DOPAC and HVA in both methamphetamine- and saline-pretreated animals, and large differences between the groups were evident at all points in time (Fig. 1). The effect of (+)-amphetamine on extracellular 5-HIAA was much smaller, but it did produce a significant gradual elevation in extracellular 5-HIAA in both groups, and both groups differed greatly at all points in time (Fig. 1).

Fig. 2 illustrates the effect of an acute injection of $(+)$-amphetamine on motor activity, and for ease of comparison, the effect of $(+)$-amphetamine on the extracellular concentration of DA is replotted from Fig. 1 as an overlay. In control animals $(+)$-amphetamine produced a typical increase in motor activity, which peaked about $60 \mathrm{~min}$ after the injection and then slowly subsided to baseline levels. (+)-Amphetamine produced a significantly more robust behavioral response in methamphetamine-pretreated animals than in controls. The onset of hyperactivity was more rapid and pronounced in methamphetamine-pretreated animals, and they were significantly more active than the controls for the first $40 \mathrm{~min}$ after the $d$-amphetamine challenge (Fig. 2).

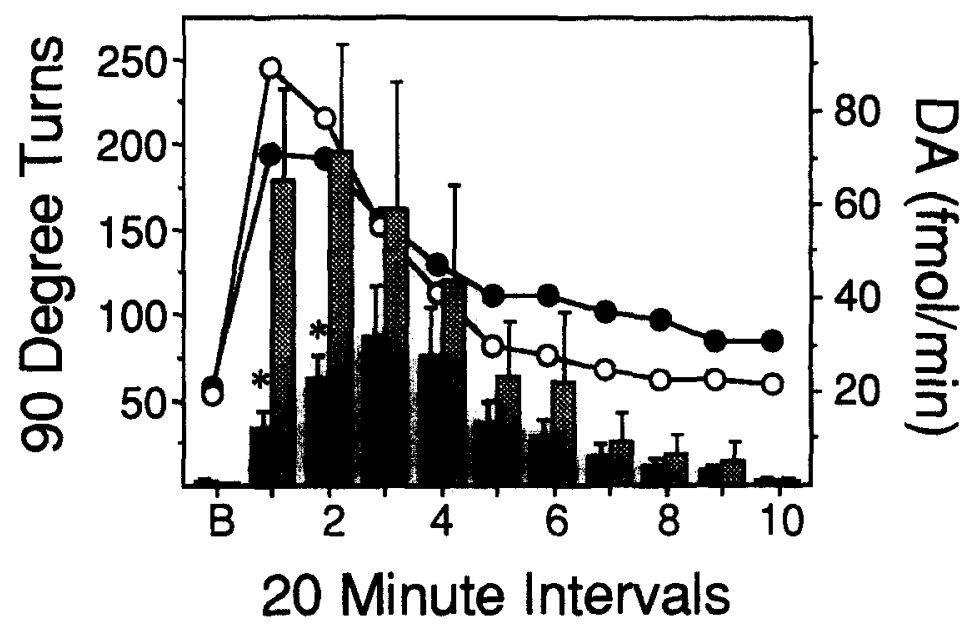

Fig. 2. The effect of a challenge injection of $(+)$-amphetamine on motor activity expressed as the average ( \pm S.E.M.) number of $90^{\circ}$ turning movements (bar graph) during a $20 \mathrm{~min}$ baseline period (B) and each of ten 20 min intervals following the amphetamine challenge. Rats were pretreated with either methamphetamine (shaded bars; $n=4$ ) or saline (open bars; $n=9$ ). Behavioral data were lost for one methamphetamine-pretreated rat and only behavioral data were obtained for two additional saline-pretreated rats. The average extracellular concentration of DA is replotted from Fig. 1 as an overlay for ease in comparing the behavioral and neurochemical data (solid symbols, methamphetamine-pretreated; open symbols, saline-pretreated). Behavior: two-way ANOVA, effect of group ( $F=3.03$, not significant), time $(F=11.9$, $P<0.001)$ and group-by-time interaction $(F=4.4, P<0.001)$. The asterisks indicate that the groups differed significantly $(P<0.05)$ during the first two 20 min intervals after $(+)$-amphetamine administration. 
In summary, repeated high doses of methamphetamine produced a persistent (at least 1 week) depletion of DA in postmortem tissue obtained from the striatum, and DA concentrations have been shown to accurately reflect DA terminal density following a lesion [2]. This lesion was not accompanied by a significant decrease in the basal extracellular concentration of DA estimated by microdialysis, and furthermore, methamphetamine-pretreated animals showed a relatively normal increase in extracellular DA in response to a $(+)$-amphetamine challenge. These data suggest that the sparing of function (i.e. absence of behavioral deficits) seen after neurotoxic doses of methamphetamine may be due to presynaptic compensatory changes in the remaining population of DA terminals that serve to 'normalize' the extracellular (and presumably synaptic) concentration of DA.

The presynaptic compensatory changes responsible for the normalization of extracellular DA after a partial lesion have not been fully characterized, but there are a number of changes that have been identified and probably interact to produce the final effect. For example, the rate of DA synthesis in the remaining terminals is known to greatly increase [17], and the discharge rate in the remaining DA cells increases [8]. This latter observation is presumably related to increased DA release from the residual DA terminals [15]. The lesion also destroys DA reuptake sites, and therefore, DA can diffuse much farther through the extracellular space in animals with a lesion than in intact animals.

It is interesting to compare the effects of $(+)$-amphetamine on extracellular DA and behavior (Fig. 2). The challenge injection of (+)-amphetamine produced a similar increase in the extracellular concentration of DA in methamphetamine- and saline-pretreated rats, but a much larger initial behavioral response in the methamphetamine-pretreated animals. It is not clear what is responsible for the behavioral effect, but it seems to be a reliable phenomenon because it has been also reported in mice given neurotoxic doses of methamphetamine [16]. Striatal DA receptor supersensitivity could be involved, but this does not develop until striatal DA is depleted by over $80-85 \%$ [15]. Another interesting possibility is that the enhanced behavioral response reflects sensitization in the nucleus accumbens. Prior amphetamine treatment is known to produce an increase in the motor stimulant effects induced by a subsequent amphetamine challenge [11], and this behavioral sensitization is accompanied by enhanced DA release in the nucleus accumbens [12]. Methamphetamine pretreatment did not deplete DA in the nucleus accumbens to the same extent as in the striatum [9], and therefore, the robust behavioral response to $(+)$-amphetamine may reflect sensitized DA release in the relatively intact nucleus accumbens.

Supported by NIDA Grant 04294 and a RCDA (NS00844) to T.E.R. We thank Laura Levy and Lori Ruddock for assistance in conducting the experiments.

1 Altar, C.A. and Marien, M.R., Preservation of dopamine release in the denervated striatum, Neurosci. Lett., 96 (1989) 329-334.

2 Altar, C.A., Marien, M.R. and Marshall, J.F., Time course of adaptations in dopamine biosynthesis, 
metabolism, and release following nigrostriatal lesions: implications for behavioral recovery from brain injury, J. Neurochem., 48 (1987) 390-399.

3 Ando, K., Johanson, C.E. and Schuster, C.R., Effects of dopaminergic agents on eye tracking before and after repeated methamphetamine, Pharmacol. Biochem. Behav., 24 (1986) 693-699.

4 Ando, K., Johanson, C.E., Seiden, L.S. and Schuster, C.R., Sensitivity changes to dopaminergic agents in fine motor control of rhesus monkeys after repeated methamphetamine administration, Pharmacol. Biochem. Behav., 22 (1985) 737.743.

5 Bittner, S.E., Wagner, G.C., Aigner, T.G. and Seiden, L.S., Effects of a high-dose treatment of methamphetamine on caudate dopamine and anorexia in rats, Pharmacol. Biochem. Behav., 14 (1981) $481-486$.

6 Castañeda, E., Whishaw, I.Q. and Robinson, T.E., Changes in extracellular dopamine concentrations measured by in vivo intracerebral dialysis after dopamine depletion, Soc. Neurosci. Abstr., 15 (1989) 559.

7 De Vito, M.J. and Wagner, G.C., Functional consequences following methamphetamine-induced neuronal damage, Psychopharmacology, 97 (1989) 432435.

8 Hollerman, J.R. and Grace, A.A.. Nigral DA cell recruitment as a compensatory mechanism, Soc. Neurosci. Abstr., 14 (1988) 1146.

9 Morgan, M.E. and Gibb. J.W.. Short-term and long-term effects of methamphetamine on biogenic amine metabolism in extra-striatal dopaminergic nuclei, Neuropharmacology, 19 (1980) 989-995.

10 Ricaurte, G.A., Schuster, C.R. and Seiden, L.S., Long-term effects of repeated methylamphetamine administration on dopamine and serotonin neurons in the rat brain: a regional study, Brain Res., 193 (1980) 153-163.

11 Robinson, T.E. and Becker, J.B., Enduring changes in brain and behavior produced by chronic amphetamine administration: a review and evaluation of animal models of amphetamine psychosis, Brain Res. Rev., 396 (1986) 157-198.

12 Robinson, T.E., Jurson, P.A., Bennett, J.A. and Bentgen, K.M., Peristent sensitization of dopamine neurotransmission in ventral striatum (nucleus accumbens) produced by past experience with (+)-amphetamine: a dialysis study in freely moving rat, Brain Res., 462 (1988) 211-222.

13 Robinson, T.E. and Whishaw, I.Q., Normalization of extracellular dopamine in striatum following recovery from a partial unilateral 6-OHDA lesion of the substantia nigra: a microdialysis study in freely moving rat, Brain Res., 450 (1988) 209-224.

14 Seiden, L.S. and Ricaurte, G.A., Neurotoxicity of methamphetamine and related drugs. In H.Y. Melzer (Eds.), Psychopharmacology: The Third Generation of Progress, Raven, New York, 1987, pp. 359366.

15 Stricker. E.M. and Zigmond, M.J., Brain monoamines, homeostasis, and adaptive behavior. In: Handbook of Physiology. Vol. 4, Intrinsic Regulatory Systems of the Brain, American Physiological Society, Bethesda, MD, 1986, pp. 677 698 .

16 Yamanaka, Y., Takano, R. and Egashira, T., Methamphetamine-induced behavioral alterations following repeated administration of methamphetamine, Jpn. J. Pharmacol., 41 (1986) 147-154.

17 Zigmond, M.J., Acheson, A.L., Stachowiak, M.K. and Stricker, E.M., Neurochemical compensation after nigrostriatal bundle injury in an animal model of preclinical parkinsonism, Arch. Neurol., 41 (1984) $856-861$. 